\title{
Single quantum dot states measured by optical modulation spectroscopy
}

\author{
N. H. Bonadeo, A. S. Lenihan, Gang Chen, J. R. Guest, and D. G. Steel \\ The Harrison M. Randall Laboratory of Physics, and The Center for Ultrafast Optical Science, \\ The University of Michigan, Ann Arbor, Michigan 48109 \\ D. Gammon, D. S. Katzer, and D. Park \\ Naval Research Laboratory, Washington DC 20375
}

(Received 13 April 1999; accepted for publication 2 August 1999)

\begin{abstract}
Using optical modulation spectroscopy, we report the direct observation of absorption lines from excitons localized in GaAs single quantum dot potentials. The data provide a measurement of the linewidth, resonance energy, and oscillator strength of the transitions, and show that states which decay primarily by nonradiative processes can be directly probed using this technique. The experiments establish this technique for the characterization of single quantum dot transitions, thereby complementing luminescence studies. (C) 1999 American Institute of Physics.
\end{abstract}

[S0003-6951(99)00939-0]

In recent years, semiconductor quantum dots (QDs) have been the subject of many scientific studies. By examining these systems, fundamental physics questions regarding the changes in optical and electronic properties arising from quantum confinement can be addressed. The quality of the QD structures has been greatly improved in the last few years using new materials and novel growing techniques. However, size variations in the confinement potential and fluctuations in the alloy concentration of the resulting QDs have complicated the use of traditional far-field spectroscopy techniques for the characterization of these samples, forcing many groups to develop high spatial resolution techniques to isolate single QDs. ${ }^{1-4}$ Much of this work has relied on photoluminescence (PL) as a probe, although other techniques such as electronic transport, ${ }^{5}$ capacitance and resonant nonlinear spectroscopy ${ }^{4}$ have been successfully employed as well. PL is attractive due to its sensitivity and relative simplicity because of the difficulty of optically probing individual QDs. However, the amount of information that can be extracted from PL is limited. For example, fully resonant probing is not possible, only luminescent states can be studied and no information about the absolute value of the oscillator strength of the transitions can be extracted. Hence, an alternative simple method that complements the luminescence studies is needed for the investigation and characterization of these quantum structures.

In this letter we report the use of optical modulation spectroscopy to directly probe the absorption lines of excitons localized in single QD. Three-dimensional confinement is achieved by lateral confinement in a quantum well $(\mathrm{QW})$ structure introduced naturally by monolayer fluctuations. Modulation spectroscopy, unlike both PL and PL excitation (PLE), allows for the measurement of the resonance frequency, oscillator strength, and linewidth of all of the optical transitions regardless of the dominant recombination processes. The measured linewidths agree very well with earlier photoluminescence ${ }^{6}$ and nonlinear spectroscopy studies ${ }^{4}$ performed in these samples. The estimated oscillator strength is $f \sim 4.2$, which is comparable to other systems. ${ }^{7,8}$ A great advantage of the optical modulation spectroscopy method is that it can be easily adapted to investigate other QD systems even in the single QD limit.
The molecular-beam epitaxy (MBE)-grown sample structure consists of a $500-\AA$-thick GaAs cap layer, followed by $5 \mathrm{GaAs}$ layers with thicknesses of $28,42,62,85$, and 142 $\AA$, respectively. These layers are separated by $250 \AA$ $\mathrm{Al}_{0.3} \mathrm{Ga}_{0.7} \mathrm{As}$ barriers and have been grown with two minute growth interruptions at the interfaces. These interruptions lead to the formation of large monolayer-high islands that are larger than the exciton Bohr radius, which effectively localize excitons in QD-like potentials. The top layer is $1000 \AA$ of GaAs and the stop etch layer is $500 \AA$ of AlAs. The substrate was removed with a liquid etch and the sample was attached to a sapphire disk to allow for transmission experiments. We focus in the present study on the $42 \AA$ layer. High spatial resolution is obtained by exciting excitons in isolated QDs through an aperture in a 100-nm-thick Al mask deposited directly on the surface of the sample. The apertures were created using e-beam lithography and metal liftoff, and range from 25 to $0.2 \mu \mathrm{m}$ in diameter. ${ }^{2}$ Figure 1(b) (top curve) shows the luminescence spectrum from a $25 \mu \mathrm{m}$ aperture which is characterized by two spectral features that correspond to the emission from excitons localized in the 15 and 16 monolayers wide regions of the sample as schematically represented in Fig. 1(a). It can be observed in Fig. 1(b) (bottom curve) that when the excitation region is reduced to 0.5

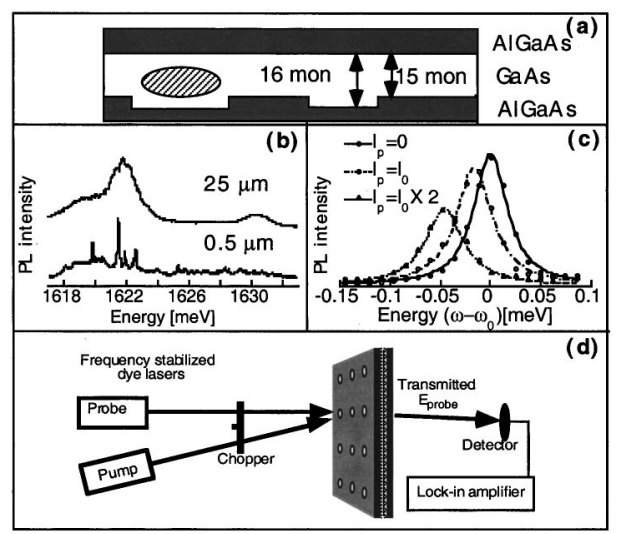

FIG. 1. (a) Diagram of the exciton confined by interface fluctuations. (b) Luminescence spectrum following the excitation of a 25 (top curve) and 0.5 $\mu \mathrm{m}$ apertures (bottom curve). (c) Luminescence spectrum from a single QD as a function of the power of the off-resonance optical field $\left(I_{0}\right.$ $\sim 100 \mathrm{~W} / \mathrm{cm}^{2}$ ). (d) A diagram of the experimental configuration. 
$\mu \mathrm{m}$, the inhomogeneously broadened lines break up into a series of sharp resonances corresponding to single QD states.

Modulation spectroscopy has been used successfully for many years to measure the transmittance and reflectance spectra of semiconductor materials and microstructures. ${ }^{9-11}$ The approach consists of applying a periodic perturbation that alters the local environment, allowing for a measurement of the derivative of the dielectric function with respect to the parameters being modified. The derivative nature of this method provides maximum sensitivity to the optical transitions permitting measurements to be made even if the interesting features are superimposed on a broad background. Studies in ensembles of nanocrystals, however, have demonstrated the difficulties associated with the determination of the homogeneous linewidth and dipole moment of the transitions from the modulated spectrum due to a broad size distribution. ${ }^{12}$ As we show in this letter, these difficulties disappear when studying a single QD.

In this sample, the single QD PL and PLE lines show a red shift and a change on oscillator strength in the presence of a nonresonant optical pump field. Figure 1(c) shows the PL spectrum for various intensities of a nonresonant field tuned to $1617.5 \mathrm{meV}$, approximately $3 \mathrm{meV}$ above $\omega_{0}$. These changes do not depend significantly on the excitation wavelength over the range of our lasers $(720-770 \mathrm{~nm})$. It was determined by using a traveling wave grating technique in an unapertured sample that the relaxation time for these changes is longer than 4 ns. ${ }^{13}$ The physical origin of these changes, is still under investigation; however, a possible explanation would be the presence of a built-in electric field across the $42 \AA$ GaAs layer, which is being modified by the diffusion of optically created charges to the surface (photovoltaic effect). This effect has been identified as the mechanism for photo modulation of the photoreflectance in bulk GaAs,${ }^{14} \mathrm{GaAs} / \mathrm{Al}_{x} \mathrm{Ga}_{1-x}$ As multiple QWs, ${ }^{15}$ and Ge. ${ }^{16}$ The built-in electric field is produced by the accumulation of charges at the surface due to pinning of the Fermi level. An alternative explanation for these changes is the thermomodulation of the resonances; however, the absence of significant line broadening, the small thickness of the sample, and the negligible difference between data collected at $6 \mathrm{~K}$ and immersed in superfluid helium make this hypothesis unlikely. Whatever the mechanism, the changes in resonance frequency and oscillator strength affect all of the states and provide a sensitive means by which to detect very weak absorption features not observable by ordinary spectroscopy.

The experimental configuration is shown in Fig. 1(d). The sample is excited through a $0.5 \mu \mathrm{m}$ diameter aperture by a nonresonant continuous-wave (cw) laser $\left(I=120 \mathrm{~W} / \mathrm{cm}^{2}\right)$ tuned to $1617.5 \mathrm{meV}$. A weaker $\mathrm{cw}$ beam $\left(I=50 \mathrm{~W} / \mathrm{cm}^{2}\right)$ transmitted through the sample probes the changes in the absorption spectrum. The wavelength of the probe beam is scanned in steps of approximately $35 \mu \mathrm{eV}$ to obtain the low resolution modulated transmission spectrum. A frequency stabilized continuously tunable cw dye laser (linewidth $<4$ $\mathrm{meV}$ ) is used to obtain the high resolution modulation spectrum of single QD states. The transmitted beam is measured by a photomultiplier tube coupled with a lock-in amplifier. We use a double modulation technique in which the pump and the probe beams are modulated at frequencies of 1 and
$1.2 \mathrm{kHz}$, respectively, and phase-sensitive detection is performed at the sum frequency. This helps to eliminate the background signal arising from the pump scattering from the apertures. The polarizations of pump and probe fields are set orthogonal further decreasing the scattered pump. The sample temperature is fixed at $6 \mathrm{~K}$. We note that the use of a laser as a probe is not necessary and could be replaced by white light source and a spectrometer (with adequate resolution).

The modulated transmittance and the luminescence spectra of a $0.5 \mu \mathrm{m}$ aperature are shown in Fig. 2. Both spectra show the typical narrow lines arising from the quantum confinement in three dimensions and while the two spectra share some common features, there are also significant differences. The modulated transmission spectrum shows a number of features not present in the PL spectrum. This is not surprising given that the transmittance spectrum is able to probe states that are dominated by nonradiative decay processes, in addition to luminescence states. An example of these nonradiative states is the resonance located at frequency $\omega_{R}$ which is absent in the PL spectrum.

A high resolution spectrum for the $\omega_{R}$ and $\omega_{S}$ resonances is shown in Figs. 3(a) and Fig. 3(b), respectively. For the analysis of the data we must take into account the nature of the electronic states being measured. For small perturbations, it has been shown that a first derivative functional form fit is appropriate for the analysis of the lineshapes in excitonic or confined systems., ${ }^{9,15,17,18}$ However, in our case, changes as small as tens of $\mu \mathrm{eV}$ in the resonant frequency represent a large portion of the resonance linewidth, therefore these changes cannot be treated perturbatively. In the low absorption limit ( $K \ll 1$, where $K$ is the absorbance), the modulated transmission is given by the difference between absorption with and without the pump beam

$$
\frac{\Delta T(E)}{T} \cong-\left[K(E)_{I=I_{0}}-K(E)_{I=0}\right],
$$

where $I$ is the pump intensity. In the case of a single QD it

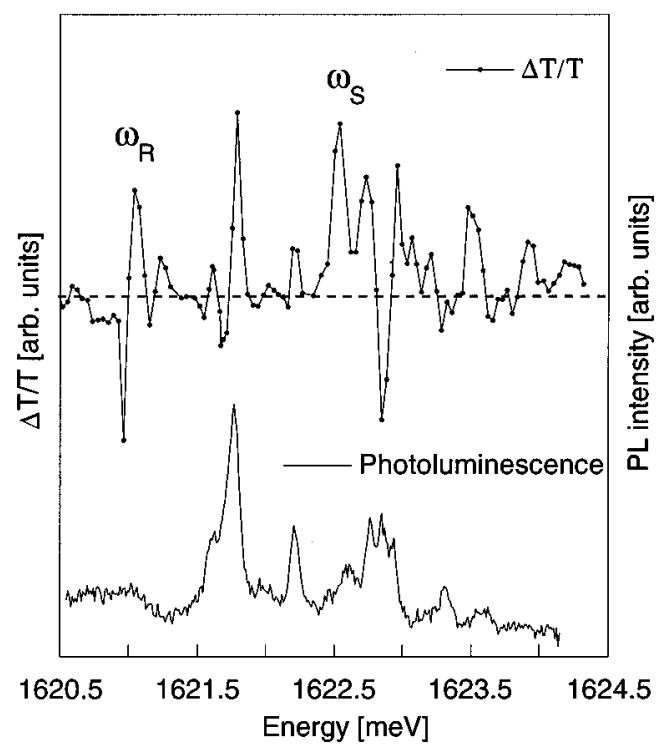

FIG. 2. A comparison of a low frequency resolution modulated spectrum and luminescence spectrum exciting through a $0.5 \mu \mathrm{m}$ aperture (laser excitation $\sim 1633 \mathrm{meV}$ ). 

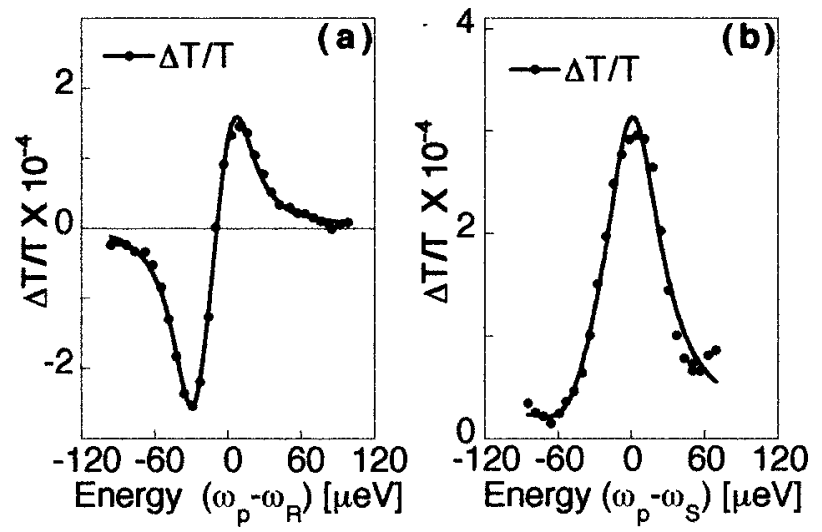

FIG. 3. A high resolution modulated transmission spectra from a single QD states located at frequencies $\omega_{R}$ (a) and $\omega_{S}$ (b) (see Fig. 2) resolves the extremely sharp lines arising from quantum confinement. The curve fits based on the functional form of Eq. (1) reveal a FWHM of 42 and $54 \mu \mathrm{eV}$, respectively.

has been shown that the lines are homogeneously broadened, ${ }^{4}$ therefore the absorption coefficient can be expressed as

$$
K(E)=\frac{f N e^{2} \hbar}{2 m_{0} \gamma_{0} n c \epsilon_{0}} \frac{1}{1+\left[\left(E-E_{0}\right) / \gamma 0\right]} .
$$

where the oscillator strength is $f$ the resonance energy is $E_{0}$ and the dephasing rate is $\gamma 0 . K_{0}=f N e^{2} \hbar / 2 \gamma_{0} n c \epsilon_{0} m_{0}$ is the unsaturated absorbance. $N$ is the areal density, which in this experiment on a single dot excited at energy $\hbar \omega_{0}$ is simply $1 / A$, where $A$ is the area of the aperture.

We fit the data plotted in Fig. 3(a) to the functional form of Eq. (1) and extract a linewidth of $2 \gamma \sim 42 \mu \mathrm{eV}$ [full width at half maximum (FWHM)], an unsaturated absorbance $K_{0}$ $\sim 2.5 \times 10^{-4}$, and an energy shift $\omega_{0}(0)-\omega_{0}(I) \sim 31 \mu \mathrm{eV}$ for that resonance. We observe that the resonance located at $\omega_{R}$ shows a lineshape characteristic of a red shift in $\omega_{0}$, typical of the quantum confined Stark effect. ${ }^{18}$ There is no change in the dephasing rate [as has been shown in Fig. 1(c)] and a small change in the oscillator strength. Using the value of $K_{0}$ we estimate the resonance oscillator strength to be $f$ $\sim 4.2$ which is comparable to the measured value for a $40 \AA$ radius $\mathrm{CuCl} \operatorname{dot}^{7}$ and the value obtained in InAs selfassembled dots. ${ }^{8}$ This value of the oscillator strength corresponds to a dipole moment $\mu \sim 10$ debye.

In contrast to the observations reported in Fig. 3(a), the lineshape plotted in Fig. 3(b) shows almost no contribution from a frequency shift which is instead dominated by a saturation of the oscillator strength of the transition. A fit using Eq. (1) gives a linewidth $2 \gamma \sim 54 \mu \mathrm{eV}$ (FWHM). This saturation could be due to the presence of an electric field parallel to the QW plane, caused either by charge migration into the QW from the barriers or by inhomogenities in the built-in electric field at the surface. It has been observed in similar systems ${ }^{19}$ that an electric field parallel to the QW reduces the oscillator strength by separating the electron and hole wave functions along the dot, thus reducing their overlap. The values of the oscillator strength obtained are very similar to the ones obtained from Fig. 3(a). It is interesting to note that even though the external perturbation has been equivalent for these QDs (i.e., the data was taken under the same experimental conditions), they have been affected in very different ways.

In summary, we have reported the observation of absorption lines corresponding to excitons localized in single QD potentials. This work takes earlier modulation spectroscopy experiments performed in ensembles of QDs to the single QD limit, allowing for a measurement of the linewidth, oscillator strength, and resonance energy of the transitions. The measured linewidths are in good agreement with both nonlinear resonant spectroscopy and luminescence measurements. In addition, we have clearly demonstrated the ability to measure states which are dominated by nonradiative decay processes. We have established this technique as a useful tool for the characterization of optical transitions in single QDs which can accelerate the search for better growth procedures and materials with which to fabricate these structures. This technique opens up the possibility of studying a new range of physical behavior such as the effects of stress (piezomodulation), temperature (thermomodulation), and electric fields (electromodulation) in these systems.

This work was supported by ARO, AFOSR, ONR, and the NSF-STC for Ultrafast Optical Science.

${ }^{1}$ K. Brunner, U. Bockelmann, G. Abstreiter, M. Walther, B. Bohm, G. Trankle, and G. Weimann, Phys. Rev. Lett. 69, 3216 (1992); H. F. Hess, E. Betzig, T. D. Harris, L. N. Pfeiffer, and K. W. West, Science 264, 1740 (1994).

${ }^{2}$ D. Gammon, E. S. Snow, and D. S. Katzer, Appl. Phys. Lett. 67, 2391 (1995).

${ }^{3}$ D. Gammon, E. S. Snow, B. V. Shanabrook, D. S. Katzer, and D. Park, Science 273, 87 (1996); S. P. Empedocles and M. G. Bawendi, Science 278, 2114 (1997); L. Landin, M. S. Miller, M.-E. Pistol, C. E. Pryor, and L. Samuelson, Science 280, 262 (1998); G. Schedelbeck, W. Wegscheider, M. Bichler, and G. Abstreiter, Science 277, 1792 (1997); K. Brunner, G. Abstreiter, G. Böhn, G. Tränkle, and G. Weimann, Phys. Rev. Lett. 73, 1138 (1994); E. Dekel, D. Gershoni, E. Ehrenfreund, D. Spektor, J. M. Garcia, and P. M. Petroff, Phys. Rev. Lett. 80, 4991 (1998); J.-Y. Marzin, J.-M. Gérard, A. Izraël, D. Barrier, and G. Bastard, Phys. Rev. Lett. 73, 716 (1994).

${ }^{4}$ N. H. Bonadeo, G. Chen, D. Gammon, D. S. Katzer, D. Park, and D. G. Steel, Phys. Rev. Lett. 81, 2759 (1998).

${ }^{5}$ L. P. Kouwenhoven, T. H. Oosterkamp, M. W. S. Danoesastro, J. Eto, D. G. Austing, T. Honda, and S. Tarucha, Science 278, 1788 (1997).

${ }^{6}$ D. Gammon, E. S. Snow, B. V. Shanabrook, D. S. Katzer, and D. Park, Phys. Rev. Lett. 76, 3005 (1996).

${ }^{7}$ T. Kataoka, T. Tozizaki, and A. Nakamura, Phys. Rev. B 48, 2815 (1993).

${ }^{8}$ R. J. Warburton, C. S. Durr, K. Karrai, J. P. Kotthaus, G. MedeirosRibeiro, and P. M. Petroff, Phys. Rev. Lett. 79, 5282 (1997).

${ }^{9}$ F. H. Pollak and H. Shen, Mater. Sci. Eng., R. 10, 275 (1993).

${ }^{10}$ O. J. Glembocki and S. V. Shanabrook, in Semiconductors and Semimet$a l s$, edited by D. G. Seiler and C. L. Litter (Academic, New York, 1992), Vol. 36, p. 221.

${ }^{11}$ M. Cardona, Modulation Spectroscopy (Academic, New York, 1969); D. E. Aspnes, in Handbook of Semiconductors, edited by M. Balkansky (North-Holland, New York, 1980), Vol. 2, p. 109.

${ }^{12}$ A. Sacra, D. J. Norris, C. B. Murray, and M. G. Bawendi, J. Chem. Phys. 103, 5236 (1995).

${ }^{13}$ A. C. Schaefer (unpublished data).

${ }^{14}$ J. L. Shay, Phys. Rev. B 2, 803 (1970).

${ }^{15}$ B. V. Shanabrook, O. J. Glembocki, and W. T. Beard, Phys. Rev. B 35, 2540 (1987).

${ }^{16}$ D. E. Aspnes, Solid State Commun. 8, 267 (1970).

${ }^{17}$ D.-S. Jiang, Y.-S. Tang, J.-B. Xia, and R. Enderlein, Superlattices Microstruct. 6, 387 (1989).

${ }^{18}$ D. A. B. Miller, D. S. Chemla, T. C. Damen, A. C. Gossard, W. Wiegmann, T. H. Wood, and C. A. Burrus, Phys. Rev. B 32, 1043 (1985).

${ }^{19}$ W. Heller, U. Bockelmann, and G. Abstreiter, Phys. Rev. B 57, 6270 (1998). 